\title{
LES LANCEURS D'ALERTE EN DROIT FRANÇAIS. IDENTITÉ, STATUT, INTERROGATIONS
}

“REPORTANTE” NO DIREITO FRANCÊS. IDENTIDADE, REGIME JURÍDICO, QUESTÕES

Mathieu Disant ${ }^{*}$

\begin{abstract}
Résumé:
Le lancement d'alerte relève d'un conflit éthique que le droit s'efforce de saisir avec difficultés. Les lanceurs d'alerte sont une figure juridique encore largement indécise. En France, un statut du lanceur d'alerte a été récemment édifié par la loi du 9 décembre 2016. Il traduit l'ambition d'unifier leur régime juridique et leur protection, tout en soulevant plusieurs interrogations. C'est ce qu'examine le présent article, achevé en mai 2018.
\end{abstract}

Mots-clés: Lanceur d'alerte. Conflit éthique. Statut. Protection. Champ d'application. Procédure de signalement.

Resumo:

A atuação do «reportante» decorre de um conflito ético que o direito se esforça para alcançar, não sem dificuldade. Os «reportantes» constituem uma figura jurídica ainda amplamente imprecisa. Na França, um estatuto do «reportante» foi recentemente construído pela Lei de 9 de dezembro de 2016. Ela expressa a ambição de englobar seu regime jurídico e sua proteção, levantando várias interrogações. É isso que o presente artigo examina, tendo sido concluído em maio de 2018.

Palavras-chave: «Reportante». Conflito ético. Regime jurídico. Proteção. Campo de aplicação. Procedimento de aviso.

Le terme "lanceur d'alerte" n'est apparu en France qu'en 1999, issu de travaux de sociologie pragmatique et d'une adaptation de la notion anglo-saxonne de whistleblower. Il a connu un véritable engouement. En France, et plus largement en Europe, chacun constate aujourd'hui la forte présence des lanceurs d'alerte sur la scène médiatique, dans le débat public et la sphère académique. Le sujet du lancement d'alerte occupe régulièrement l'actualité et suscite un intérêt grandissant auprès du grand public, où la fascination se mêle aux interrogations. De nombreux travaux, à l'origine en sociologie puis en droit, ont déjà été consacrés aux lanceurs d'alerte. ${ }^{1}$ Ils ont permis de

\footnotetext{
* Agrégé des Facultés de droit (Full Professor). Professeur à l'Université Lyon Saint-Etienne (Jean Monnet). Directeur du Centre de recherches critiques sur le droit (UMR CNRS 5137). Professeur invité à l'Université de São Paulo.

1 Nous nous permettons de renvoyer à l'ouvrage que nous avons dirigé: M. Disant et D. Pollet-Panoussis, Les lanceurs d'alerte, LGDJ-Lextenso, 2017, 424 pages. Il propose une analyse transdisciplinaire, différents aspects du droit français et des analyses étrangères. Parmi elles, nous tenons à signaler la contribution de notre collègue et ami le Professeur Fernando Menezes (USP) qui a pu faire profiter les lecteurs francophones 
souligner comment le droit s'en est progressivement saisi, le cas échéant de faire utilement état du droit comparé et d'exprimer les craintes ou réticences au regard des implications juridiques plus ou moins maîtrisées.

Je ferai immédiatement une observation liminaire pour souligner l'importance, sur ces sujets, que l'étude juridique soit ouverte à la connaissance interdisciplinaire. Comprendre les ressorts du processus de lancement d'alerte et son rôle contemporain requiert d'être attentif aux analyses historique, économique et sociologique, mais aussi d'en saisir les mobilisations et caractéristiques du point de vue éthique. Tous ces regards sont indispensables à la compréhension du droit qui se fait. Cela ne se résume donc pas à l'étude $d u$ droit mais mobilise aussi l'analyse sur le droit. C'est également indispensable pour se projeter sur les usages et l'efficacité des procédures mises en place en matière de protection.

Les lanceurs d'alerte ont leurs figures symboliques. Hier, Deltour(Luxleaks), Manning ("War-logs"), Snowden (sans doute le plus influent et emblématique) et, en France, Frachon, ${ }^{2}$ Vasseur... ${ }^{3}$ Aujourd'hui, Christopher Wylie dans l'affaire "FacebookCambridge Analytica", du nom de l'entreprise accusée d'avoir utilisé les données de millions d'individus pour manipuler les élections et construire "l'Alt-Right" aux ÉtatsUnis - faisant apparaître aux yeux de tous combien, devant la gestion non maîtrisée des données personnelles, la frontière entre la recherche marketing et l'espionnage se trouble. Le souci taxonomique conduit à distinguer le groupe des "Leakers" (ce qu'on qualifierait en français de fuiteurs), sorte de dénonciateurs à grande échelle, en prenant conscience que le "leak" est devenu une véritable marque, dans tout ce que cela recouvre. Il confère à l'alerte une connotation industrielle peu enviable.

Mais il n'y a pas que ces grands dossiers mondiaux, ces données massives et cas emblématiques. Il y a aussi des centaines de personnes qui, dans leur quotidien, sont susceptibles de rencontrer un dilemme professionnel: mettre en péril (voire sacrifier) leur carrière ou faire une alerte sur un fait grave et d'intérêt général, qu'il s'agisse d'un crime, d'un délit ou d'un risque grave de sécurité, santé et environnement. Il faut insister sur cette situation de dissonance cognitive - pour emprunter le langage de psychologie sociale - pour comprendre l'inconfort psychologique dans laquelle se situe le (véritable) lanceur d'alerte et qui, à certains égards, est une condition de sa reconnaissance juridique en cette qualité.

d'une analyse émergente au Brésil.

2 Irène Frachon est un médecin pneumologue qui, en poste dans un centre hospitalier, a joué un rôle de lanceur d'alerte dans l'affaire dite du «médiator», médicament qui aurait provoqué la mort d'au moins deux mille malades.

3 Véronique Vasseur, médecin-chef d'une prison, a dénoncé dans un livre retentissant les conditions carcérales indignes dont elle a été témoin. 
Les lanceurs d'alerte se retrouvent tiraillés entre des valeurs contradictoires et soumises à des injonctions paradoxales (dont l'injonction éthique fait partie). Il leur faut évaluer des éléments conflictuels, c'est la nature même du lancement d'alerte. Sont ainsi mis en balance:

- l'intérêt public du signalement confronté à l'obligation de confidentialité,

- le dommage causé à l'employeur et le devoir de loyauté à l'égard de l'organisation face à l'intérêt public,

- $\quad$ les représailles qu'il va subir comme lanceur d'alerte $v s$. la difficulté de vivre avec la connaissance d'un danger non révélé.

La ventilation des canaux de signalement - nous y reviendrons - est un moyen d'adapter la procédure du lancement d'alerte à ces tensions en fonction de la situation donnée.

Évidemment, le lancement d'alerte est aussi à replacer dans un conflit éthique. D'une part, lancer une alerte revient à remettre en cause sa hiérarchie au sein de l'organisation, laquelle est par hypothèse complice par action ou par omission. D'autre part, le chemin que va emprunter le lanceur d'alerte pour dénoncer des faits entre en conflit avec les règles et procédures de l'organisation (même si une procédure interne est prévue) et, plus largement, avec la solidarité de groupe et tout un univers symbolique. Les travaux en sciences de l'éthique établissent bien les difficultés liées à cette transgression.

Le problème se pose donc potentiellement au citoyen ordinaire, ce qui attise l'enjeu de le protéger. En contrepoint, il faut demeurer prudent à l'égard de l'idéal, naïf ou onirique, tendant à envisager tous les signalements comme du lancement d'alerte et tous les lanceurs d'alerte comme des justiciers solitaires ou des héros ordinaires, ce qui fausse, par un a priori de valeur, l'analyse sur le statut des lanceurs d'alerte.

Le sujet n'est pas tout à fait consensuel. Les lanceurs d'alerte sont des "vigies citoyennes" pour certains, "traîtres" ou "délateurs" pour d'autres. Ils sont au cœur de la question éthique et soulèvent des difficultés juridiques qui ne sont pas nouvelles. Il suffit de penser à la figure antique du sycophante, sorte de statut juridique originel du délateur. Aristophane y voyait un risque de dissolution du Démos et d'affaiblissement $\mathrm{du}$ pouvoir populaire. Démosthène leur reprochait explicitement de flatter le peuple et d'entretenir une rhétorique populiste. En outre, dans l'histoire contemporaine française, particulièrement en raison de la seconde guerre mondiale, les signalements contraires à la loi ont été inconsciemment assimilés à de la délation; cela a entretenu une confusion qui a ralenti la mise en place d'une protection par l'Etat (elle n'a débuté qu'en 2007).

A vrai dire, sur le plan du droit positif, en France, les lanceurs d'alerte forment une figure juridique indécise dont les contours demeurent mal maîtrisés. Sans doute parce qu'elle manifeste une évolution, souvent analysée mais rarement clarifiée, de 
la perception du Droit et du rapport à la règle. Comment l'alerte est-elle saisie par le droit? Comment le droit appréhende-t-il, dans leur diversité, celles et ceux qui "donnent un coup de sifflet" pour reprendre la signification du terme anglo-saxon de whistleblower, ce qu' on traduirait plus volontiers dans le langage populaire français comme "tirer la sonnette d'alarme". Comment s'opère le signalement des manquements en vue de faire cesser des agissements pouvant représenter un risque pour autrui? Cela pose des questions juridiques fondamentales: peut-on être condamné lorsqu'on révèle une vérité? Comment concevoir l'excuse d'intérêt général? Bref, quelle(s) identité(s) juridique(s) pour ces lanceurs? Quelle reconnaissance juridique? Quelle protection par le droit? Quelles concrétisations par le juge et autorités compétentes? Mais aussi quelles limites?

Ces questions ont pris un relief particulier en raison de l'édification récente d'un statut du lanceur d'alerte, qui fait ressortir l'ambition d'unifier le régime juridique.

Avant de l'analyser, je tiens à souligner qu'il y a plusieurs façons complémentaires d'aborder le sujet.

On peut l'envisager au travers les rapports complexes que nouent l'alerte et le risque (risques pour la santé, l'environnement, les finances publiques, les libertés individuelles...). Le lanceur d'alerte encourt lui-même un certain nombre de risques, singulièrement en cas d'alerte jugée abusive. La structure où se déploie l'alerte (État, entreprise...) n'y échappe pas non plus.

On peut aussi analyser l'alerte comme l'un des objets d'étude des interactions entre la science, la société et la démocratie. ${ }^{4}$ Il est certain que le lanceur d'alerte incarne une nouvelle figure en et $d u$ droit aujourd'hui à l'œuvre dans les démocraties occidentales. L'alerte ouvre, à cet égard, un champ d'étude important sur la décision en situation de controverses.

On pourrait aussi souligner la dimension historico-culturelle sous-jacente. Par exemple, l'étude de la situation américaine, pays pionnier en matière de whistleblowing, permet de relever le développement d'une culture de l'alerte dans le secteur de l'entreprise suite aux scandales financiers dans les années 1990, parmi lesquels l'affaire Enron. Il faut dire, qu'aux Etats-Unis, dès 1863 le False Claims Act encourageait le signalement des fraudes à l'encontre de l'État fédéral. Cette culture s'est d'ailleurs diffusée dans le monde entier (y compris en France) par l'application extraterritoriale de la législation, par exemple le Sarbanes Oxley Act (SOX) adopté le 30 juillet 2002.

Mais on peut encore tenter de cerner les contours du lanceur d'alerte en l'observant dans ses différents états, dans ses différentes situations. Car il y a des identités très diverses de ce qu'on appelle "lanceur d'alerte" dans le discours juridique. Ce qui

En dernier lieu, LECLERC, Olivier. Protéger les lanceurs d'alerte: la démocratie technique à l'épreuve de la loi. Issy-les-Moulineaux: LGDJ - Lextenso, 2017. (Exégèses). 
témoigne de la difficulté, lorsqu'il s'agit d'examiner comment le droit saisit l'éthique individuelle, à en faire émerger une catégorie juridique saisissable. Ce qui renforce, d'autant, le besoin d'une certaine mise en ordre.

\section{L'identité du lanceur d'alerte en droit français}

1.1. Qu'est-ce qu'un lanceur d'alerte? Il faut avoir conscience qu'il s'agit d'une question difficile, et que la définition donnée en droit positif - sur lequel je vais revenir pour la France - est doublement relative.

D’une part, elle n'empêche pas que se développe cette étiquette sur le plan médiatico-sociétal, où est lanceur d'alerte celui qui s'affirme comme tel et reconnu par la collectivité. C'est d'ailleurs assez révélateur qu'on attribue ce qualificatif à Assange. Il y a une évidente attractivité de l'appellation "lanceur d'alerte" dont il est facile de se trouver auréolé.

D'autre part, il faut tenir compte des spécificités des droits nationaux. Edward Snowden, Bradley/Chelsea Manning (lourdement condamné), et d'autres, ne sont pas des lanceurs d'alerte pour les juridictions de leur pays. Et ce même si le lancement a des effets internationaux. On observe toutefois qu'une relative standardisation est à l'œuvre, particulièrement en Europe. Même si les législations européennes sont encore hétérogènes, il y une harmonisation sous l'influence d'une recommandation du Conseil de l'Europe en 2014 et un projet de directive européenne en cours vise à poser une législation horizontale. La Cour européenne des droits de l'homme poursuit, quant à elle, sa politique jurisprudentielle sur le terrain de l'entrave à la liberté d'expression, dans ses multiples et différents aspects. ${ }^{5}$

1.2. De façon traditionnelle, à partir de catégories juridiques à peu près établies, on a pu dissocier deux types de lanceur d'alerte, selon qu'on puisse le qualifier de dénonciateur légal ou le considérer comme un citoyen désobéissant.

Dans la première hypothèse, la dénonciation est fondée sur des systèmes d'alerte ou de signalements d'illégalités, de risques ou de comportements contraires à la déontologie professionnelle (discrimination, harcèlement). L'alerte est alors reconnue légitime par le législateur: plus qu'un droit, elle peut être une obligation. C'est le cas, par exemple, en application de l'article 40 du Code de procédure pénale qui prévoit l'obligation de dénonciation au Procureur de la République des crimes et délits dont les agents publics ont connaissance dans l'exercice de leurs fonctions. C'est le cas aussi dans

Récemment, sur l'atteinte excessive à la liberté d'expression d'un conseiller municipal lorsqu'il «signale les préoccupations [des électeurs] et défend leurs intérêts»: CEDH, 7 septembre 2017, Lacroix c/ France, req. N. 41519/12. 
le cadre des dispositifs d'alerte éthique et sociale dans les entreprises, dans le cadre du droit du travail. Cette forme d'alerte est présente dans le domaine de la lutte contre la corruption, des conflits d'intérêts, crimes et délits, risques sociaux, environnementaux ou sanitaires.

Dans la seconde hypothèse, l'alerte se présente comme un acte citoyen: l'individu transgresse délibérément une norme au nom de sa conscience personnelle et du souci subjectif de se conformer à un principe supérieur d'intérêt général. L'alerte "citoyenne" - que l'on assimile couramment à "l'alerte éthique" par un tour de force sémantique - n'est alors pas sans lien avec la désobéissance civile. ${ }^{6}$ Le lanceur d'alerte prend ici clairement le risque de s'exposer à des sanctions, notamment disciplinaires et/ ou pénales.

Cette distinction appelle deux observations.

D'abord, il faut avoir conscience qu'elle est en partie artificielle. Une forme d'alerte peut en cacher une autre, la seconder voire la devancer. Il n'est pas rare qu'un scandale environnemental, sanitaire ou financier soit à l'origine d'une évolution consacrant un dispositif légal d'alerte. C'est le cas dans l'affaire du Mediator dénoncée par Irène Frachon, à l'origine de l'adoption de la loi n. 2011-2012 du 29 décembre 2011 relative au renforcement de la sécurité sanitaire du médicament et des produits de santé. Le terme "lanceur" y prend tout son sens d'initiateur, à l'origine aussi de l'évolution du droit. Ce n'est pas rien lorsqu'on observe la propension, dans notre société et notre droit, à ce que des scandales soient des coups d'accélérateur pour la production de la norme juridique.

Ensuite, cette distinction - dénonciateur légal / acte citoyen - est fortement relativisée depuis la consécration, en droit positif français, d'un statut "global" ou unifié du lanceur d'alerte. Ce statut résulte de la loi n. 2016-1691 du 9 décembre 2016 relative à la transparence, à la lutte contre la corruption et à la modernisation de la vie économique, dite "loi Sapin 2". Elle "institutionnalise" le lancement d'alerte et retient une définition assez large du lanceur, auquel elle reconnait une protection.

1.3. J'ajouterai un élément qui me paraît important. En réalité, les situations que l'on évoque sous le "lancement d'alerte" sont souvent différentes et mélangées. Il faut être attentif à la dimension fonctionnelle ou finaliste. Le lanceur d'alerte découvre les éléments à son corps défendant et fait un signalement pour mettre fin à un dysfonctionnement. C'est son but. C'est la raison d'être du lancement. C'est un point de différence avec le "leaker" qui s'épanouit dans la dissémination de l'information au service d'une cause: il sert une idéologie de la transparence absolue et n'est, à cette fin,

LOCHAK, D. «L'alerte éthique, entre dénonciation et désobéissance». AJDA, Paris, n. 39, 2014. p. 2.236. 
qu'un agent de communication ou de destruction. Ce n'est pas l'état du lanceur d'alerte qui se reconnait par une stratégie, à la fois éthique et judiciaire, toute entière tournée vers un objectif immédiat et identifié: arrêter le dysfonctionnement. La diffusion au public n'est alors qu'un dernier recours; pour un lanceur d'alerte, le "leak" (la fuite) est un moyen ultime et non une fin.

Les "leaks" posent inévitablement la question du relai des médias, de leur rôle et du risque de manipulation. Nous sommes dans une société alertée dans laquelle les journalistes sont dans une situation antithétique. Par tradition, ils connaissaient et protégeaient l'anonymat de leurs sources (qu'on songe à l'affaire du Watergate et la fameuse "gorge profonde"). Aujourd'hui, les journalistes reçoivent des "leaks" mais n'ont bien souvent aucune idée d'où cela vient - ni a fortiori les motivations de ces distributeurs d'information en masse. C'est un véritable circuit de blanchiment d'informations - on l'a vu avec Assange - qui rend aveugle sur l'origine, les ressorts et les objectifs de ces informations. Il s'agit là d'un combat éthique et d'un enjeu pratique de taille, à la mesure de la fonction constitutionnelle des médias et des divers auxiliaires de la société de l'information qui doivent assurer ce filtre. Pour le relever, il faut tenir l'idée que le lanceur d'alerte n'est pas un agitateur politique et que son statut s'oppose à la logique d'agitation et de malveillance. Au demeurant, il est important que les lanceurs d'alerte ne soient pas eux-mêmes manipulés - le vérifier est d'ailleurs le premier réflexe des associations de défense des lanceurs d'alerte: identifier l'infraction, les éléments de preuve de la démarche, la réalité des représailles, etc. En somme, il s'agit de s'assurer que l'alerte est responsable.

\section{La protection des lanceurs d'alerte par le droit français}

Je ferai une série d'observations sur ce point, en laissant plusieurs aspects sectoriels de côté (selon les domaines, les difficultés rencontrées et les procédures applicables seront différentes).

L'analyse du droit français est marquée par l'intervention de la récente loi “Sapin 2" du 9 décembre 2016 précitée, pleinement applicable depuis le 1 er janvier 2018. Cette loi appelle plusieurs observations.

2.1. $1^{\text {ère }}$ observation. Le législateur a entendu dépasser l'approche fragmentée jusqu'alors applicable en matière de protection des lanceurs d'alerte pour élaborer un statut. Avant la loi de 2016, plusieurs textes législatifs s'étaient additionnés. La jurisprudence était aussi intervenue. ${ }^{7}$ Une législation d'ensemble, plus protectrice, a

Quelques mois avant l'adoption de la loi de 2016, en s'appuyant sur le droit d'expression au sens de l'article 10-1 de la Convention européenne des droits de l'homme, la Cour de cassation instaura une protection contre 
ainsi succédé au millefeuille législatif qui s'était constitué depuis $2007 .{ }^{8}$ La France s'est largement inspirée du précédent britannique, là où un dispositif global de protection des lanceurs d'alerte s'est imposé dès 1998 au Royaume-Uni ${ }^{9}$ et en 2014 en Irlande. ${ }^{10}$ Le régime juridique du lanceur d'alerte prétend ainsi à la généralisation en dépassant une stricte approche sectorielle.

On aurait tort de présenter systématiquement cela comme étant, en soi, un progrès en termes de simplification. La globalisation du régime s'accompagne de flottements potentiellement contre-productifs. Par exemple, le problème des modalités de reconnaissance de la qualité de lanceur d'alerte reste entier puisque, dans le système retenu depuis 2016, elle ne repose que sur un principe d'auto-déclaration. De ce point de vue purement technique, il n'est pas interdit d'y voir un recul par rapport à la loi "Blandin" de 2013 en matière d'alerte sanitaire et environnementale. Par ailleurs, on voit déjà se fissurer la portée englobante de la définition à l'aune, dernièrement, de celle retenue dans les ordonnances "travail": en tant qu'exception au plafonnement des indemnités prud'homales, le licenciement pour cause d'alerte est restreint à l'alerte sur un crime ou un délit.

Quoi qu'il en soit, l'affirmation d'un régime global ne veut pas dire qu'il faille négliger les spécificités sectorielles. De fait, l'alerte éthique, eu égard à son objet, continue de jouer un rôle essentiel dans certains secteurs de la vie publique, tels que la protection de l'environnement ou la préservation des finances publiques et la probité fiscale à l'aune du statut d'aviseur. Rôle tout aussi singulier dans la vie des affaires, où les risques de dévoiement de l'alerte rémunérée accompagnent la nécessité de prendre en considération le secret des affaires et accords de confidentialité. En outre, les textes ont maintenu ou mis en place des régimes spéciaux d'alerte, avec des règles procédurales particulières en matière de signalement, notamment pour la banque et l'assurance, la

le licenciement du lanceur d'alerte ayant signalé, de bonne foi, des conduites ou actes illicites constatés sur le lieu de travail: Cass. soc., 30 juin 2016, n. 15-10.557.

8 Notamment la loi n. 2007-1598 du 13 novembre 2007 relative à la lutte contre la corruption, au $J O$ du 14 novembre 2007, p. 18648 (transposition de la Convention « mérida »); la loi n. 2011-2012 du 29 décembre 2011 relative au renforcement de la sécurité sanitaire du médicament et des produits de santé, au JO du 30 décembre 2011, p. 22667; la loi n. 2013-316 du 16 avril 2013 relative à l'indépendance de l'expertise en matière de santé et d'environnement et à la protection des lanceurs d'alerte, au $J O$ du 17 avril 2013, p. 6465; la loi n. 2013-907 du 11 octobre 2013 relative à la transparence de la vie publique, au $J O$ du 12 octobre 2013, p. 16829; la loi n. 2013-1117 du 6 décembre 2013 relative à la lutte contre la fraude fiscale et la grande délinquance économique et financière, au $J O$ du 7 décembre 2013, p. 19941; loi 2016-483 du 20 avril 2016 relative à la déontologie et aux droits et obligations des fonctionnaires, au $J O$ du 21 avril 2016.

9 Il s'agit du PIDA adopté en 1998: Public Interest Disclosure Act. Le Royaume-Uni a été le premier État européen à se doter d'un dispositif global de protection des lanceurs d'alerte. Par la suite, la Roumanie (2004), la Slovénie (2010), le Luxembourg (2011) et l'Irlande (2014) l'ont imité.

10 Il s'agit du PDA adopté en 2014: Protected Disclosures Act. 
sécurité aérienne, l'environnement ou le domaine du renseignement, en raison de la sensibilité et de la complexité de ces domaines.

2.2. $2^{\text {ème }}$ observation. La loi consacre désormais, dans son article 6 , une définition générale du lanceur d'alerte comme désignant

\begin{abstract}
"toute personne physique qui révèle ou signale, de manière désintéressée et de bonne foi, un crime ou un délit, une violation grave et manifeste d'un engagement international régulièrement ratifié ou approuvé par la France, d'un acte unilatéral d'une organisation internationale pris sur le fondement d'un tel engagement, de la loi ou du règlement, ou une menace ou un préjudice graves pour l'intérêt général, dont elle a eu personnellement connaissance".
\end{abstract}

À cette définition est associée une protection à vocation générale, applicable quel que soit le domaine de l'alerte.

Le champ d'application du dispositif mérite deux précisions.

D’une part, sont écartés a contrario les signalements effectués pas les associations, syndicats, ordres professionnels ou tout autre collectif. Ces structures sont contenues au seul rôle d'appui dans les réalités diverses du terrain.

D'autre part, si la loi française retient une définition large du lanceur, déconnectée a priori du cadre professionnel et de la qualité de travailleur à la différence de nombreuses autres législations nationales (telles que celles applicables au RoyaumeUni ou en Irlande), les canaux d'alerte hiérarchisés prévus par la loi "Sapin" ne sont applicables que dans le contexte d'une relation de travail, comme l'a expressément relevé le Conseil constitutionnel dans sa décision n. 2016-741 DC du 8 décembre 2016. La procédure de signalement, telle que séquencée par la loi dans son article 8, s'adresse aux salariés ou aux agents de l'organisme qui fait l'objet de l'alerte, le cas échéant aux soustraitants ou aux collaborateurs occasionnels de ce dernier. De fait, le lanceur d'alerte est, dans la très grande majorité des cas, un agent public ou un salarié.

On ajoutera que la définition retenue pose la difficulté, pour celui qui en revendique le bénéfice, de devoir émettre, tantôt une appréciation pénale des faits dont il a connaissance, tantôt la double qualification d'une menace grave à l'intérêt général. C'est un biais redoutable. Il pourrait bien être source de désillusions pour ceux qui, sans forcément le pouvoir ni le vouloir, et sans filet, se retrouvent dans la posture de l'homme de loi, voire du procureur ou de l'autorité publique.

2.3. $3^{\text {ème }}$ observation. La loi Sapin 2 oblige les entreprises et les administrations d'au moins 50 salariés, ainsi que les collectivités de plus de 10000 habitants, à mettre en place un dispositif d'alerte interne pour faire remonter les signalements des lanceurs d'alerte, qu'ils soient salariés ou collaborateurs extérieurs. 
La procédure interne s'articule en trois étapes. La première est qualifiable au sens strict d"'alerte interne" et repose sur un principe d'épuisement des voies de recours internes: le lanceur d'alerte doit saisir tout supérieur hiérarchique direct ou indirect, ou un référent désigné par l'employeur. C'est en principe le rôle du référent déontologue, au nom d'une logique de guichet unique posée en avril 2017. Sur ce point, de nombreuses difficultés sont d'ores et déjà apparues en termes de recrutement, périmètre, moyens, compétences matérielles et territoriales... Elles sont consubstantielles à la logique attrapetout dans laquelle est placé ce référent et à la grande latitude laissée par les textes qui risque de mener à des pratiques très différentes. Au cas précis, il demeure des interrogations pratiques, par exemple en cas de divergence d'appréciation entre le chef de service et le référent déontologue, ce dernier ne disposant d'aucun pouvoir sur le premier. En outre, s'il peut exister un référent specialis alerte éthique (on parle de "référent éthique"), ${ }^{11}$ on peut alors s'interroger quant à l'articulation des compétences avec le référent déontologue, ou sur la possibilité de mutualisation du référent éthique entre plusieurs collectivités qui pose des questions pratiques (externalisation? dématérialisation?) non résolues par le décret $\mathrm{n}$. 2017-564 du 19 avril 2017.

Quoi qu'il en soit, si l'alerte n'a pas été traitée dans un délai raisonnable (ce standard demeurant flou faute de délai de réponse établi), le salarié passe à la deuxième étape: s'adresser à la justice ou à une autorité administrative (préfet, inspections des services, agences administratives, etc.). Enfin, si son signalement reste sans effet dans un délai de trois mois, il peut le rendre public auprès des médias, des ONG ou des syndicats. A ce jour, l'ONG Transparency international France semble mobilisé autour d'une vingtaine de cas.

«En cas de danger grave et imminent, ou en présence d'un risque de dommages irréversibles», ou si sa hiérarchie est impliquée, le lanceur d'alerte peut passer directement à la deuxième ou à la troisième étape. Il faut bien souligner que, dans ce cas, il risque de perdre les protections légales s'il ne dispose pas de preuves suffisantes, ce qui soulève une nouvelle fois la difficulté, pour le lanceur d'alerte, d'être en mesure d'évaluer et de qualifier correctement la situation qu'il observe.

On aura compris que, pour bénéficier de la protection, le lanceur d'alerte doit respecter les conditions du lancement d'alerte tant sur le fond (action désintéressée et de bonne foi) que sur la forme (respect de la procédure hiérarchisée de recueil et traitement de l'alerte). Alors, le lanceur bénéficie d'une stricte confidentialité de son identité et des informations qu'il révèle, le cas échéant d'un fait justificatif du délit de violation du secret

C'est une obligation au sein des collectivités publiques de plus de 10000 habitants; soit, de fait, 1000 communes concernées. 
professionnel, ${ }^{12}$ et d'une protection contre les représailles professionnelles (notamment avec la possibilité de saisine en référé du Conseil de prud'hommes en cas de licenciement suite au signalement d'une alerte). Le champ d'application et la portée de la protection demeurent toutefois sujets à interrogation notamment s'agissant de la levée des poursuites pénales. Par ailleurs, une incrimination a été insérée à l'encontre de ceux qui, d'une façon ou d'une autre, feraient obstacle au signalement. Si le destinataire de l'alerte divulgue l'identité du lanceur d'alerte, les personnes mises en cause ou les informations signalées, il encourt une peine de deux ans de prison et 30000 euros d'amende.

Il convient d'évoquer, sur la protection, la dimension européenne. Il en ressort des "zones grises" d'alerte sans protection, consécutives au déboulement conceptuel de la notion en droits européens. L'émergence de la réglementation européenne constitue un progrès certain mais n'est pas sans imperfections ni incertitudes. Une avancée significative est en cours. La Commission européenne porte un projet de directive européenne pour les lanceurs d'alerte. Ce projet a été dévoilé publiquement le 23 avril 2018. Il est largement influencé par des organisations de la société civile du secteur, et très proche d'un rapport d'initiative n. 2016/2055 adopté par le Parlement européen en octobre 2017. Ce projet tend à poser un standard minimal de protection au sein de l'Union, sur la base d'une généralisation du système de signalement interne accompagné d'une clause de sauvegarde générale, en ayant en vue le déficit de protection dans certains pays tels la Hongrie ou la Pologne. Un temps évoquée, la possibilité que tout pays européens puisse offrir asile à tout lanceur d'alerte persécuté reste posée.

2.4. $4^{\text {ème }}$ observation. Le salarié n'est pas contraint de suivre une procédure interne. Il peut choisir de saisir directement le procureur de la République. Il peut aussi s'adresser au défenseur des droits, chargé, non de traiter une alerte (il n'est pas habilité à constater les dysfonctionnements à l'origine du signalement ni ne peut aucunement effectuer de vérification en ce sens), mais d'orienter et de protéger de lanceur.

Le dispositif de protection fait ainsi intervenir le Défenseur des droits. Pour une part, il s'agit d'un prolongement, à peu près maîtrisé, des compétences traditionnelles de cette autorité, en ce que les mesures de rétorsions et représailles dont peut souffrir le lanceur d'alerte sont attraites dans le champ des discriminations. L'innovation tient surtout à ce que le Défenseur des droits a désormais la charge d'une nouvelle mission d'assistance et d'orientation des lanceurs d'alerte, obligation peu définie et dont la portée soulève plusieurs interrogations pratiques. Cela résulte en partie de l'urgence avec laquelle ont été introduits par voie d'amendement les dispositions concernées de la loi "Sapin 2" et, sans

$12 \quad$ Nouvel article 122-9 du code pénal. Précisons que les faits ou informations couverts par le secret de la défense nationale, le secret médical ou le secret des relations entre avocat et son client ne peuvent faire l'objet d'une alerte. 
étude d'impact, menés les travaux en commission. De fait, le Défenseur des droits a publié un "guide d'orientation et de protection des lanceurs d'alerte" qui, à travers une série de fiches très pédagogiques, s'adresse aux personnes souhaitant effectuer un signalement. L'idée consiste, à terme, à mettre en place un portail chargé, le cas échéant, de transmettre les alertes aux autorités compétentes.

Il convient de rappeler que la version initiale du dispositif ouvrait au Défenseur des droits la faculté d'apporter un soutien financier aux lanceurs d'alerte, sans autres critères clairs, au risque d'une dérive institutionnel aux effets incertains. Il nous semble heureux que ce volet du dispositif ait été censuré par le Conseil constitutionnel dans sa décision n. 2016-740 DC précitée. En tant qu'autorité administrative indépendante, dont le législateur français s'est par ailleurs efforcé de rationaliser l'identité dans un statut général par les lois du 20 janvier 2017, 1'autorité du Défenseur des droits demeure fondée sur l'indépendance, la neutralité et l'impartialité du processus décisionnel. Cela est difficilement compatible, en droit comme en fait, avec une fonction de "financeur" il n'est d'ailleurs pas certain que cela le soit définitivement et en tous points avec celle d"'orientateur" - tant le cumul de ces types de compétences serait allé à l'encontre des règles qui président à son fonctionnement, y compris au plan du statut contentieux des actes pris par le Défenseur des droits, sans même évoquer le problème au regard des droits constitutionnel et conventionnel de la défense.

Il n'est pas simple d'élaborer des règles juridiques qui puissent, dans des limites raisonnables tout en étant efficaces, se saisir des questions éthiques. Le dispositif français de protection des lanceurs d'alerte n'est sans doute pas parfait. Il a le mérite d'exister et de favoriser la discussion au niveau européen. Il faudra évaluer, sur la durée, non seulement son efficacité dans les différents univers de travail, mais surtout sa contribution à la démarche asymptotique de la démocratie.

France, 7 mai 2018.

\section{Références}

DISANT, Mathieu; POLLET-PANOUSSIS, Delphine (dir.). Les lanceurs d'alerte. Issy-lesMoulineaux: LGDJ - Lextenso, 2017.

FRANCE. Loi n. 2007-1598 du 13 novembre 2007 relative à la lutte contre la corruption. JORF, Paris, n. 264, p. 18.648, nov. 2007. Disponible sur internet: https://www.legifrance.gouv.fr/ affichTexte.do?cidTexte=JORFTEXT000000524023\&categorieLien=id. 
FRANCE. Loi n. 2011-2012 du 29 décembre 2011 relative au renforcement de la sécurité sanitaire du médicament et des produits de santé. JORF, Paris, n. 0302, p. 22.667, dec. 2011. Disponible sur internet: https://www.legifrance.gouv.fr/affichTexte.do?cidTexte=JORFTEXT000025053440\&cate gorieLien=id\%23JORFARTI000025053734.

FRANCE. Loi n. 2013-316 du 16 avril 2013 relative à l'indépendance de l'expertise en matière de santé et d'environnement et à la protection des lanceurs d'alerte. JORF, Paris, n. 0090, p. 6.465, avril 2013. Disponible sur internet: https://www.legifrance.gouv.fr/affichTexte.do?cidTexte=JORF TEXT000027324252\&categorieLien=id.

FRANCE. Loi n. 2013-907 du 11 octobre 2013 relative à la transparence de la vie publique. JORF, Paris, n. 0238, p. 16.829, oct. 2013. Disponible sur internet: https://www.legifrance.gouv.fr/ affichTexte.do?cidTexte=JORFTEXT000028056315\&categorieLien=id.

FRANCE. Loi n. 2013-1117 du 6 décembre 2013 relative à la lutte contre la fraude fiscale et la grande délinquance économique et financière. JORF, Paris, n. 0284, p. 19.941, dec. 2013. Disponible sur internet: https://www.legifrance.gouv.fr/affichTexte.do?cidTexte=JORFTEXT000028278976\&cate gorieLien=id.

FRANCE. Loi n. 2016-483 du 20 avril 2016 relative à la déontologie et aux droits et obligations des fonctionnaires. JORF, Paris, n. 0094, avril 2016. Disponible sur internet: https://www.legifrance. gouv.fr/affichTexte.do?cidTexte=JORFTEXT000032433852\&categorieLien=id.

LECLERC, Olivier. Protéger les lanceurs d'alerte: la démocratie technique à l'épreuve de la loi. Issy-les-Moulineaux: LGDJ - Lextenso, 2017. (Exégèses).

LOCHAK, Danièle. L'alerte éthique entre dénonciation et désobéissance. AJDA - L'Actualité Juridique, Droit Administratif, Paris, n. 39, p. 2.236-2.241, 2014.

UNITED KINGDOM. Public Interest Disclosure Act 1998. Disponible sur internet: http://www. legislation.gov.uk/ukpga/1998/23/contents. 
